\title{
CENT'ANNI DI POESIA ITALIANA
}

\author{
G. D. Leoni \\ da Universidade Católica de São Paulo e da \\ Universidade Mackenzie
}

Il periodo della moderna poesia italiana dovrebbe logicamente chiudersi dentro il ventennio (1920-1940) limitato dalle due guerre mondiali: purtroppo il frequente ripetersi di spaventosi avvenimenti ci ha abituati a usarli anche come divisioni didattiche di critica letteraria. Ma per ragioni storiche ed estetiche non mi sembra posssibile accennare in breve ai motivi dominanti di questa poesia senza richiamarmi a poeti e a idee del periodo precedente; anzi, è necessario partire proprio dal maggior poeta che ha dominato e chiuso il secolo scorso, per poter seguire le diverse tappe e giungere ad una conclusione basata su constatazioni obiettive che non debbono mancare nello spirito di chi in terra straniera é chiamato a illustrare aspetti della propria letteratura. Tuttavia, un'analisi storico-estetica porta sempre con sè una interpretazione soggettiva che determina valutazioni e preferenze particolari nell'animo dello studioso piú onesto; e con maggior ragione nell'animo di uno studioso che è anch'esso personaggio, oltre che spettatore, d'un periodo letterario ancora aperto: è impossibile fare la storia di noi stessi, poiché ci manca la giustizia inesorabile del tempo. In ogni modo, cercherò almeno di esporre con chiarezza alcune osservazioni sostanziali, lasciando in chi $\mathrm{mi}$ legge la libertà di sintetizzare i risultati di questa rapida rassegna.

n volume che raccoglie in poco piú di mille pagine tutta la produzione poetica di Giosue Carducci, ha un sottotitolo molto significativo: "1850-1900". Mezzo secolo di poesia, che 
si svolge a poco a poco e sempre piú si irrobustisce nella forma e nel contenuto, passando dai voli giovanili agli scatti della ribellione, dalla forza della sana maturità alla serena operosa vecchiezza. E un arco luminoso che proietta una viva, iridescente, anelante gioia di lavoro, compiuto con onesta passione di uomo e di studioso. L'uomo fu uguale al maestro, lo studioso non fu minore del poeta. Si è detto e ripetuto che il Carducci fu un poeta ribelle, in eterna lotta contro il romanticismo; ed è vero, se per romanticismo intendiamo la poesia sdolcinata degli eterni sognatori. Ma saremmo in errore escludendo dal Carducci quella parte di sano romanticismo ch'è in tutti noi, amanti della bellezza e dell'azione della pienezza della vita. Il merito maggiore del Carducci sta appunto qui, nell'aver ridato un sano sentimentalismo alla vera poesia, quella cioè che esce dal cuore commosso e dall'animo acceso a nobili visioni. Proprio all'inizio del suo ultimo volume di poesie ("Rime e Ritmi") egli metteva alcuni versi dedicati ad una fanciulla:

O piccola Maria, di versi a te che importa?

Esce la poesia, o piccola Maria, quando malinconia batte del cor la porta.

O piccola Maria, di versi a te che importa?

Che il poeta polemista dei 'Giambi ed Epodi", il classico poeta delle "Odi barbare", il cantore delle glorie patrie e della serena bellezza ellenica abbia voluto ricordare e trascrivere questi semplici versi accanto alle composizioni dense di storia e di cultura, è per me segno non indubbio di avvertimento e di ammonimento. Per il Carducci la poesia fu segnacolo di lotta e dolcezza di imagini, come in quel congedo alle "Rime nuove" dove meravigliosamente scolpí il poeta nel grande artiere, che dopo aver forgiato spade e scudi, serti e diademi, tabernacoli e altari, tripodi e vasi ricchi di lavoro ,per sé fa uno strale d'oro, e lo lancia contro il sole: "Guarda come in alto ascenda - e risplenda, - guarda e gode, - e piú non 
vuole". Ma la poesia del Carducci, appunto per questo suo disinteresse sentimentale, acquista un indiscutibile valore di duplice potenza: da un lato apre le porte ad un classicismo non piú ristretto alla latinità, ma ampio nei secoli e nei luoghi, dalle rive del Tevere a quelle dell'Tlisso; dall'altro lato fa di questa enorme tradizione le basi per l'avvenire della patria. Poesia storica, dunque, delle leggende eroiche e delle memorie, nelle quali si rinnova di generazione in generazione la coscienza nazionale dei popoli;; ma poesia storica che, passando da Roma, centro di una concezione di vita umanamente forte e civilizzatrice, all'armonia di un panteismo cosmico, diventa poesia universale. Su questa via lo avevano preceduto l'Alfieri e il Foscolo; eppure ciò che dà alla poesia carducciana una caratteristica originale inconfondibile è la schiettezza con la quale affronta la realtà per ridurla a ideale: egli è un istintivo che nella sua opera progredisce incessantemente, pur tra reazioni piú o meno appariscenti, fino ad arrivare a quella serena energia, che sarà il jiú mirabile testamento spirituale trasmesso alle molte generazioni di discepoli.

Eppure, se escludiamo tre continuatori del grande poeta - Enrico Panzacchi, Severino Ferrari e Giovanni Marradi, gli altri, dopo un inizio carducciano, si preoccuparono sopratutto di apparire troppo "carducciani" e fecero di tutto per non esserlo. ì il caso di Giovanni Pascoli, che nel 1892 pubblicava la sua prima raccolta di versi. L'ondata di decadentismo simbolista, venuta dalla Francia, aveva travolto anche questo giovane poeta; ma egli si salva rifugiandosi nel classicismo che è tuttavia ben diverso da quello del Carducci. Un profondo senso di religiosità, che va dalla pietà umana al dolore che è anch'esso forza, sembra gravare sempre sulla poesia del Pascoli: sono frammenti di una intima confessione; sono richiami al passato; sono voci che dal passato ritornano alla realtà quotidiana; sono incanti che la fantasia suscita e il dolore conforta; sono tremole luci di una grande fiamma, che riassume famiglia, patria, umanità; sono facili commozioni di un fanciullo, anch'esso istintivo, ma piú ingenuo, quasi sbalordito di fronte al mistero della vita. Questo misticismo fatto di lagrime e di bontà, ha suggerito al Pascoli una poesia 
che spesso si trastulla nel particolare e che rimane sempre indefinita nella suggestione: una poesia dettata dal "fanciullino" che è in noi - è una sua teoria estetica, - il quale sa tante cose a cui di solito noi non badiamo e che noi non potremmo né pensare né ridire. Vita e poesia si compenetrano tanto fortemente, che la eterna antinomia della nostra coscienza diventa un'indagine piena di ansia, di incertezza, di sogno, cosi come nei versi si manifesta per mezzo di spezzettature fragili, di incisi titubanti, di gridi soffocati, di ricordi affiorati su dal mondo dei morti. Di fronte alla poesia del futuro, che nel Carducci balza dalla gioiosa forza e dal fecondo lavoro, la poesia del Pascoli si attarda nella contemplazione del passato, tra una lagrima di rimpianto e una pietosa dolcezza di conforto umanitario.

Lo stesso decadentismo che allontanava il Pascoli dal Carducci, si manifestava quasi contemporaneamente in Gabriele D'Annunzio, ma sotto forma ben diversa. Il poeta, che col Carducci era diventato "artiere" e col Pascoli "consolatore", col D'Annunzio diventa "eroe". Alle visioni il Pascoli ha dato un'anima, il D'Annunzio una sensazione; e tutta sensazioni è stata la sua vita e la sua poesia, quella foggiata al "vivere inimitabile", questa ad una ricchezza verbale, che spesso rapisce e stordisce. Colore e suono concretano i canti dannunziani con un vigore insolito che giungono all'esaltazione: "I miei canti sono prole - delle foreste, - altri dell'onde, - altri delle arene, - altri del Sole, - altri del vento Argeste". Il poeta dà alle sue parole una natura terrestre, marina, celeste; e gode della loro melodia (che troppo spesso è una melodia da dizionario), come noi godiamo sulla spiaggia ascoltando la monotona e pur sempre diversa voce del mare. Del Pascoli è bello il frammento: accadde ad un critico di affermare giustamente che quanto piú breve è il frammento pascoliano, tanto piú è artistico (affermazione pericolosa, poiché si giungerebbe all'assurdo di definire perfetta la poesia... non composta dal poeta). Del D'Annuzio è bella la parola; tanto che se il tempo sta già sfrondando gran parte dell'opera dannunziana, a lui rimarrà eterno il merito di aver arricchito la lingua italia- 
na forse ancor piú del Manzoni. Ma ciò che piú nuoce a questa poesia non è la magniloquente verbosità o la esuberante armonia dei colori o la sinfonica melodia dei ritmi: è l'esser vissuta soltanto col suo geniale creatore, poiché solo lui ha potuto vivere in modo eroico una vita che superava la realta del presente. $E$ con lui si è spenta.

facile immaginare come in un ambiente artistico cosi minato dal decadentismo (che soltanto nature geniali, come quelle del Pascoli e del D'Annunzio, potevano superare e che invece stava affogando tutta la letteratura europea) sia nata la scuola dei dannunziani. Sarebbe troppo ironico affermare che da un brutto verso del D'Annunzio - e anche $i$ grandi poeti possono anche cadere nella sciatteria - è nata la poesia del "novecento"; ciò che è vero è che da lui ha preso ardire una vasta schiera di poeti, o meglio di verseggiatori, i quali dal D'Annunzio derivavano un trasmodato soggettivismo e una libertà che superava i confini dell'arte. Ciò che prima era umanità sociale e religiosa, compresa nel suo illimitato panteismo cosmico, si frantumava nel lirismo intimo, nella effusione sentimentale dell'autobiografia, nel languido atteggiamento di una superiorità spirituale che giunge fino alla soddisfazione di non essere compresi. Col decadere della morale poetica decade vertiginosamente l'equilibrio artistico della lingua: quella ricchezza di vocabolario che è una gloria del D'Annunzio (anche se è stato necessario compilare un grosso vocabolario "dannunziano" senza l'uso del quale molte volte la sua poesia diventa incomprensibile), si è disintegrata nella rinuncia alla purezza linguistica, anzi nella rinuncia alla sintassi, alla grammatica e perfino all'equilibrio logico di una pagina tipografica...

Confesso che è per me penoso ricordare come durante alcuni anni tanti scrittori abbiano perduto ogni controllo artistico: mania di novità, mania di reazione, esasperazione antitradizionale, meccanico riflesso della vita rapidissima, sintesi fisica delle sensazioni, grido di lotta che si perde in simboli. Il futurismo di Filippo Tommaso Marinetti e dei suoi seguaci ha tentato di distruggere il passato. Tentativo ardito, poiché la tradizione letteraria italiana ha due millenni; tentativo fal- 
lito, poiché in arte reagire vuol dire abbattere e anche costruire. $E$ il futurismo ha costruito castelli in aria, creando soltanto il misero ambiente dei "geni incompresi", una folla di impotenze artistiche, che riversavano nella parola la loro ignoranza o la loro smodata buffoneria. L'unica attenuante che possiamo concedere a questo modernismo è quella stessa chè la critica ha finalmente dovuto ammettere per il marinismo secentesco: non è un movimento italiano, ma europeo, che non dà origine ai vari marinismi d'oltr'Alpe, ma che si sviluppa contemporaneamente ad essi, nati da un' atmosfera sparsa sull'intero continente. Anche il futurismo e le sue sottospecie (poesia pura, ermetica, ecc.) sono una ventata di follia che dalla Francia si sparge sull'Italia e trova il suo ambiente preparato dall'ultimo dannunzianesimo, ormai capitato fra le mani di ragazzi capricciosi e viziati. Gli ermetici, poi, hanno dato il tracollo: possono essere ricordati come la piú bassa scala del futurismo, perché almeno nei futuristi c'era un impeto, sia pur trasmodato, di vita, di azione, di lotta; gli ermetici sono dei rassegnati e dei vinti per definizione, poiché il voler essere incompresi o è insoddisfazione spirituale o morboso egoismo, quando addirittura non è altro che insufficienza artistica. In ogni modo, il poeta ermetico rinuncia all'unica gioia del poeta - e in questa gioia dobbiamo mettere anche il dovere altruistico - di dare e di non chiedere. Ha ragione quel critico, il quale afferma che, nel leggere una poesia ermetica, il lettore si sente piú poeta di chi l'ha scritta...

Eppure, proprio durante i primi accenni della scuola dannunziana, per quella forza tradizionale che non viene mai meno allo spirito italiano anche nei momenti piú tragici, una piccolaschiera di poeti assisteva quasi impassibile alla ventata di follia artistica che stava per sconvolgere l'eterna belezza della poesia. La critica, scorgendo in questi poeti solitari una caratteristica comune - una specie di sfumato sentimento, che sembra quasi la nebbia delle prime luci dell'alba o degli ultimi bagliori del tramonto - ha dato loro il nome di "crepuscolari"; invero, erano dei pascoliani in ritardo, che non sapevano raggiungere le vette del misticismo o dell'umanitarismo, e si accontentavano di un modesto e semplice senso 
poetico della vita, di un raccoglimento spirituale tra la realtà quotidiana e il sogno malinconico, senza impeti, senza clamori, senza rimpianti. La loro piccola esperienza bastava; bastava la coscienza di una crisi intima, sincera, coraggiosa anche nella rinuncia. Questa tragedia "crepuscolare", che è in tutti noi della generazione del 1900, trovava i suoi poeti in Sergio Corazzini, in Marino Moretti, in Aldo Palazzeschi, in Guido Gozzano, nella prima fase poetica di Arturo Onofri. Essi erano come fanciulli abbagliati dalla fantasmagoria dannunziana $o$ dai fuochi d'artificio del futurismo; ma si ritraevano a poco a poco nel loro animo riflessivo e commosso, lirico e narrativo, spesso ironico, spesso infantíle, ma sempre sincero. Nell'apparenza erano dei modernisti; nell'animo erano forzatamente dei tradizionalisti. Il loro stile semplice, disadorno, quasi prosastico li riconduceva senza accorgersene alle immagini pascoliane, al Pascoli provinciale (forse il migliore); e indicavano che in mezzo a tanto trambusto il cammino della buona poesia non era scomparso.

Infatti, il buon cammino esisteva, sempre pronto ad accogliere una poesia piú modesta, ma piú sana. Vittorio Locchi - l'unico poeta uscito dalla prima guerra mondiale - dava l'esempio; e l'esempio, sulle orme di qualche solitario (Adolfo de Bosis, Giovanni Bertacchi...) fruttificava: Angiolo Orvieto, Diego Valeri, Vittore Vittori, Ada Negri (rinnovata dopo le avventure moderniste) riconducevano la poesia italiana ai ritmi e alla logica chiarezza di un sano sentimento lirico. A poco a poco la materia si fa piú densa nel suo contenuto spirituale: Luigi Orsini ritrova le voci della sua terra e della patria, Francesco Chiesa oscilla nell'ansia di rinnovare nel futuro la forza e la dolcezza del passato, Francesco Pastonchi cesella odi e sonetti, Giuseppe Lipparini ritorna al sensualismo pagano, Angiolo Silvio Novaro indaga nell'affetto i dolori della vita, Vincenzo Gerace riprende la tecnica tradizionale e classicheggiante, Ugo Betti concreta la sua virile rassegnazione in uno spirito tormentato, ma sano. I nomi non contano piú e un arido elenco sarebbe un inutile sfoggio di modesta erudizione. Quel che conta è che la poesia italiana ha ritrovato se stessa; e se ancora oscilla nella ricerca di un poeta che sappia elevarsi al di sopra di tutti per continuare la gloria del grande trino- 
mio (Carducci, Pascoli, D’Annunzio), ciò dipende dai tempi, non certo adatti ad una esatta rinascenza dello spirito, o dalla logica cecità critica di un osservatore contemporaneo. Tutto fa credere, tuttavia, che, dopo le esperienze temporanee del poeta-eroe o del poeta-consolatore, risorgerá dal rinnovamento la gloriosa figura del poeta-artiere.

Con questa immagine carducciana chiudiamo i brevi accenni sugli aspetti della moderna poesia italiana. Non sembri mania di schematismo didattico se oso racchiudere in un ampio cerchio le mie rapide immagini su cent'anni di poesia: partendo dal Carducci, avanzando col Pascoli e poi col D'Annunzio, continuando coi dannunziani e tutte le loro sottospecie di futuristi, simbolisti e ermetici, sono risalito coi crepuscolari al Pascoli e ho riabbracciato i poeti che col Carducci risentono l'amore per il ritmo non meno che la dignità artistica. Nella rinnovata missione del poeta-artiere è da aspettarsi una rinnovata coscienza dei principi nazionali, che furono guida al Carducci per rafforzare l'unità ideale di una grande Italia. Anche noi godiamo di questa speranza: per la patria non invano palpitano i cuori degli assenti come non invano in ogni secolo alla patria hanno giovato i canti dei veri poeti. 